\title{
Chain Rules for Higher Derivatives
}

\author{
H.-N. Huang, S. A. M. Marcantognini and N. J. Young
}

September 23, 2005

We define a notion of higher-order directional derivative of a smooth function and use it to establish three simple formulae for the $n$th derivative of the composition of two functions. These three "higher-order chain rules" are alternatives to the classical Faà di Bruno formula. They are less explicit than Faà di Bruno's formula, but are much simpler and avoid Diophantine or combinatorial complications.

\section{Introduction}

In principle it is easy to calculate a higher derivative of the composition $f \circ g$ of two sufficiently differentiable functions $f$ and $g$ : one can simply apply the "chain rule"

$$
(f \circ g)^{\prime}=\left(f^{\prime} \circ g\right) g^{\prime}
$$

as many times as needed. For example,

$$
\begin{aligned}
(f \circ g)^{\prime \prime} & =\left(\left(f^{\prime} \circ g\right) g^{\prime}\right)^{\prime}=\left(f^{\prime} \circ g\right)^{\prime} g^{\prime}+\left(f^{\prime} \circ g\right) g^{\prime \prime} \\
& =\left(f^{\prime \prime} \circ g\right)\left(g^{\prime}\right)^{2}+\left(f^{\prime} \circ g\right) g^{\prime \prime} .
\end{aligned}
$$

By continuing in this vein we can readily obtain an expression for any particular higher order derivative of $f \circ g$ in terms of derivatives of $f$ and $g$. Formulae for $(f \circ g)^{(n)}$, for an arbitrary positive integer $n$, have been known since the 19th century: an admirable scholarly article by Warren P. Johnson [8] not only gives several such formulae but also describes the history of the problem. Of all the formulae the most popular is named after Francesco Faà di Bruno ${ }^{1}$, who published it in 1855 $[4,5]$, though according to Johnson [8, p. 228], the result had been anticipated by

\footnotetext{
${ }^{1} \mathrm{~A}$ remarkable man who not only wrote an influential treatise on binary forms ("Théorie des formes binaires" (Paris, 1876), translated into German (Leipzig, 1881)) but was also declared Saint of the Catholic church for his charitable and religious works [5].
} 
J.F.C. Tiburce Abadie in 1850. The formula states that

$$
\begin{aligned}
& \frac{d^{n}}{d t^{n}} f(g(t))= \\
& \quad \sum \frac{n !}{k_{1} ! k_{2} ! \cdots k_{n} !} f^{(k)}(g(t))\left(\frac{g^{\prime}(t)}{1 !}\right)^{k_{1}}\left(\frac{g^{\prime \prime}(t)}{2 !}\right)^{k_{2}} \cdots\left(\frac{g^{(n)}(t)}{n !}\right)^{k_{n}},
\end{aligned}
$$

where the sum is over all nonnegative integer solutions of the Diophantine equation $k_{1}+2 k_{2}+\cdots+n k_{n}=n$, and $k:=k_{1}+k_{2}+\cdots+k_{n}$. For example, in the case $n=3$, we find that the nonnegative integer solutions of the equation

$$
k_{1}+2 k_{2}+3 k_{3}=3
$$

are $\left(k_{1}, k_{2}, k_{3}\right)=(0,0,1)$, or $(1,1,0)$ or $(3,0,0)$ with corresponding values of $k$ being 1,2 , or 3 , respectively. Faà di Bruno's formula (1) then gives

$$
\begin{aligned}
\frac{d^{3}}{d t^{3}} f(g(t))= & \frac{3 !}{0 ! 0 ! 1 !} f^{\prime}(g(t))\left(\frac{g^{\prime \prime \prime}(t)}{3 !}\right)+\frac{3 !}{1 ! 1 ! 0 !} f^{\prime \prime}(g(t))\left(\frac{g^{\prime}(t)}{1 !}\right)\left(\frac{g^{\prime \prime}(t)}{2 !}\right) \\
& +\frac{3 !}{3 ! 0 ! 0 !} f^{\prime \prime \prime}(g(t))\left(\frac{g^{\prime}(t)}{1 !}\right)^{3} \\
= & f^{\prime}(g(t)) g^{\prime \prime \prime}(t)+3 f^{\prime \prime}(g(t)) g^{\prime}(t) g^{\prime \prime}(t)+f^{\prime \prime \prime}(g(t))\left(g^{\prime}(t)\right)^{3} .
\end{aligned}
$$

Faà di Bruno's formula, and others given in [8], have a substantial combinatorial aspect; a less combinatorial and generally more transparent treatment is that of Spindler [11].We shall present an alternative formula for $(f \circ g)^{(n)}$ which differs from Faà di Bruno's in two ways: it makes use of higher order directional derivatives, and it avoids combinatorial or Diophantine questions. It is surprising if anything new can be said about such a classical topic, but we have not found anything similar in [8] or other recent papers $[2,9,11]$. We needed the formula for the study of the spectral Carathéodory- Fejér problem: given $k \times k$ matrices $V_{0}, V_{1}, \ldots, V_{n}$, determine whether there exists an analytic matricial function $F$ in the unit disc $\mathbb{D}$ such that

- the eigenvalues of $F(\lambda)$ lie in $\mathbb{D}$ for all $\lambda \in \mathbb{D}$, and

- $F^{(j)}(0)=V_{j}, \quad 0 \leq j \leq n$.

We may derive a necessary condition with the aid of a higher chain rule. If such a function $F$ exists then we may consider the function

$$
f(z, \lambda)=\operatorname{det}(z I-F(\lambda))
$$

This $f$ is analytic in $\lambda$ on $\mathbb{D}$, and for fixed $\lambda$ is a polynomial in $z$ whose roots lie in $\mathbb{D}$. Moreover, $\partial^{j} f /\left.\partial \lambda^{j}\right|_{\lambda=0}$ for $j=0,1, \ldots, n$ can be found explicitly in terms of the $V_{j}$ by the chain rule in Theorem 2 below. One of the main results in [6] states that, subject to a genericity condition, the existence of a function $f(z, \lambda)$ with the 
appropriate properties is also sufficient for the solvability of a spectral CarathéodoryFejér problem.

We give the formula in three slightly different versions, two for the case that $g$ is a function of one variable and a third for more general $\mathrm{g}$.

\section{Directional derivatives and higher chain rules}

Let $X$ and $Y$ be real or complex Banach spaces, let $\Omega$ be an open subset of $X$ and let $f: \Omega \rightarrow Y$ be Fréchet-differentiable. (In the case that $X$ and $Y$ are Euclidean spaces the notion of Fréchet differentiability coincides with the usual notion of differentiability from real analysis. The definition for the general case can be found in [7, Section 5.2] and [10, Chapter 29].) For any $x_{0} \in \Omega$ and non-zero $x_{1} \in X$, the directional or Gateau derivative of $f$ at $x_{0}$ along $x_{1}$ is defined to be

$$
\lim _{z \rightarrow 0} \frac{1}{z}\left[f\left(x_{0}+z x_{1}\right)-f\left(x_{0}\right)\right] .
$$

This is an element of $Y$, sometimes denoted by

$$
D_{x_{1}} f\left(x_{0}\right) \quad \text { or } \quad \frac{d}{d x_{1}} f\left(x_{0}\right) .
$$

The limit exists, since $f$ is supposed Fréchet differentiable: if $D f\left(x_{0}\right) \in \mathcal{L}(X, Y)$ is the Fréchet derivative of $f$ at $x_{0}$ then, for any $x_{1} \in X$,

$$
\frac{d}{d x_{1}} f\left(x_{0}\right)=D f\left(x_{0}\right) x_{1} ;
$$

here $\mathcal{L}(X, Y)$ denotes the space of bounded linear operators from $X$ to $Y$. The reader is referred to [7, Section 5.2] and [10, Chapter 29] for further details.

We shall introduce notation for higher-order directional derivatives. As far as we know, this is a new notion.

Definition 1 Let $X, Y$ be real or complex Banach spaces, let $\Omega$ be an open set in $X$ and let $f: \Omega \rightarrow Y$ be $n$ times Fréchet differentiable. We define, for $j=0,1, \ldots, n-1$,

$$
\Delta_{j} f: \Omega \times X^{j} \rightarrow Y
$$

inductively for $x_{0} \in \Omega, x_{1}, x_{2}, \ldots, x_{n} \in X$ by

$$
\begin{aligned}
& \Delta_{0} f\left(x_{0}\right)=f\left(x_{0}\right), \\
& \Delta_{j+1} f\left(x_{0}, x_{1}, \ldots, x_{j+1}\right)=\frac{d}{d\left(x_{1}, x_{2}, \ldots, x_{j+1}\right)} \Delta_{j} f\left(x_{0}, x_{1}, \ldots, x_{j}\right) .
\end{aligned}
$$

Thus

$$
\begin{array}{r}
\Delta_{j+1} f\left(x_{0}, x_{1}, \ldots, x_{j+1}\right)=\lim _{z \rightarrow 0} \frac{1}{z}\left[\Delta_{j} f\left(x_{0}+z x_{1}, x_{1}+z x_{2}, \ldots, x_{j}+z x_{j+1}\right)\right. \\
\left.-\Delta_{j} f\left(x_{0}, x_{1}, \ldots, x_{j}\right)\right] \\
=\left.\frac{\partial}{\partial z} \Delta_{j} f\left(x_{0}+z x_{1}, x_{1}+z x_{2}, \ldots, x_{j}+z x_{j+1}\right)\right|_{z=0},
\end{array}
$$


and we may write

$$
\Delta_{n} f\left(x_{0}, x_{1}, \ldots, x_{n}\right)=\frac{d}{d\left(x_{1}, \ldots, x_{n}\right)} \frac{d}{d\left(x_{1}, \ldots, x_{n-1}\right)} \cdots \frac{d}{d\left(x_{1}, x_{2}\right)} \frac{d}{d x_{1}} f\left(x_{0}\right) .
$$

Example 1 - the exponential function.

Directly from Definition 1 we find, for $x_{j} \in \mathbb{C}$,

$$
\begin{aligned}
\Delta_{1} \exp \left(x_{0}, x_{1}\right) & =\left.\frac{\partial}{\partial z} e^{x_{0}+z x_{1}}\right|_{z=0}=e^{x_{0}} x_{1}, \\
\Delta_{2} \exp \left(x_{0}, x_{1}, x_{2}\right) & =\left.\frac{\partial}{\partial z} e^{x_{0}+z x_{1}}\left(x_{1}+z x_{2}\right)\right|_{z=0}=e^{x_{0}}\left(x_{1}^{2}+x_{2}\right), \\
\Delta_{3} \exp \left(x_{0}, x_{1}, x_{2}, x_{3}\right) & =\left.\frac{\partial}{\partial z} e^{x_{0}+z x_{1}}\left[\left(x_{1}+z x_{2}\right)^{2}+x_{2}+z x_{3}\right]\right|_{z=0} \\
& =e^{x_{0}}\left(x_{1}^{3}+3 x_{1} x_{2}+x_{3}\right) .
\end{aligned}
$$

We regard $\Delta_{n} f\left(x_{0}, \ldots, x_{n}\right)$ as the directional derivative of $f$ of order $n$ at $x_{0}$ in the direction $\left(x_{1}, \ldots, x_{n}\right)$. There is another natural interpretation of the appellation " $n$th order directional derivative of $f$ ", to wit the function

$$
\Delta^{n} f: \Omega \times X^{2^{n}-1} \rightarrow Y
$$

defined inductively by

$$
\Delta^{1} f=\Delta_{1} f, \quad \Delta^{n+1} f=\Delta_{1}\left(\Delta^{n} f\right) .
$$

There is a close connection between $\Delta_{n}$ and $\Delta^{n}$.

Theorem 1 Let $X, Y$ be Banach spaces, let $\Omega$ be an open subset of $X$ and let $f$ : $\Omega \rightarrow Y$ be $n$ times continuously Fréchet differentiable. For $m \in \mathbb{N}$, let

$$
L_{m}: \Omega \times X^{m} \rightarrow \Omega \times X^{2^{m}-1}
$$

be defined inductively by

$$
\begin{aligned}
& L_{1}\left(x_{0}, x_{1}\right)=\left(x_{0}, x_{1}\right), \\
& L_{m+1}\left(x_{0}, x_{1}, \ldots, x_{m+1}\right)=\left(L_{m}\left(x_{0}, \ldots, x_{m}\right) ; L_{m}\left(x_{1}, \ldots, x_{m+1}\right)\right),
\end{aligned}
$$

for $x_{0} \in \Omega$ and $x_{j} \in X, j \geq 1$. Then

$$
\Delta_{n} f=\left(\Delta^{n} f\right) \circ L_{n}: \Omega \times X^{n} \rightarrow Y .
$$

Thus

$$
\begin{aligned}
& \Delta_{2} f\left(x_{0}, x_{1}, x_{2}\right)=\Delta^{2} f\left(x_{0}, x_{1}, x_{1}, x_{2}\right), \\
& \Delta_{3} f\left(x_{0}, x_{1}, x_{2}, x_{3}\right)=\Delta^{3} f\left(x_{0}, x_{1}, x_{1}, x_{2}, x_{1}, x_{2}, x_{2}, x_{3}\right) .
\end{aligned}
$$

$\Delta_{n} f$ has the merit of a relative economy in the number of its arguments, and earns its keep by virtue of the following three higher-order chain rules. The first two concern functions $f \circ g$ where $g$ is a function of a single real or complex variable. Let $\mathbb{K}$ denote either $\mathbb{R}$ or $\mathbb{C}$. 
Theorem 2 Let $X, Y$ be Banach spaces over $\mathbb{K}$ and let $\Psi, \Omega$ be open subsets of $\mathbb{K}, X$ respectively. Let

$$
g: \Psi \rightarrow \Omega, \quad f: \Omega \rightarrow Y
$$

have $n$ continuous Fréchet derivatives, for some $n \in \mathbb{N}$. For any $t \in \Psi$,

$$
(f \circ g)^{(n)}(t)=\Delta_{n} f\left(g(t), g^{\prime}(t), \ldots, g^{(n)}(t)\right) .
$$

Proof. In the case $n=1$ we have

$$
\begin{aligned}
\Delta_{1} f\left(g(t), g^{\prime}(t)\right) & =\left.\frac{\partial}{\partial z} f\left(g(t)+z g^{\prime}(t)\right)\right|_{z=0} \\
& =D f(g(t))\left(g^{\prime}(t)\right) \\
& =(f \circ g)^{\prime}(t)
\end{aligned}
$$

by the classical chain rule.

Suppose the assertion holds for some $j<n$, that is, for any $t \in \Psi$,

$$
\frac{d^{j}}{d t^{j}} f \circ g(t)=\Delta_{j} f\left(g(t), g^{\prime}(t), \ldots, g^{(j)}(t)\right) .
$$

Then, for $t \in \Psi$,

$$
\begin{aligned}
\frac{d^{j+1}}{d t^{j+1}} f \circ g(t) & =\frac{d^{j}}{d t^{j}}(f \circ g)^{\prime}(t) \\
& =\frac{d^{j}}{d t^{j}} \Delta_{1} f\left(g(t), g^{\prime}(t)\right) \\
& =\frac{\partial^{j}}{\partial t^{j}}\left(\left.\frac{\partial}{\partial z} f\left(g(t)+z g^{\prime}(t)\right)\right|_{z=0}\right) \\
& =\left.\frac{\partial^{j}}{\partial t^{j}} \frac{\partial}{\partial z} f\left(g(t)+z g^{\prime}(t)\right)\right|_{z=0} .
\end{aligned}
$$

The function $(t, z) \mapsto f\left(g(t)+z g^{\prime}(t)\right)$ has $j+1$ continuous derivatives on an open set in $\mathbb{K}^{2}$, and so the partial derivatives can be interchanged to give

$$
\frac{d^{j+1}}{d t^{j+1}} f \circ g(t)=\left.\frac{\partial}{\partial z} \frac{\partial^{j}}{\partial t^{j}} f\left(g(t)+z g^{\prime}(t)\right)\right|_{z=0} .
$$

Hence, by the inductive hypothesis,

$$
\begin{aligned}
\frac{d^{j+1}}{d t^{j+1}} f \circ g(t) & =\left.\frac{\partial}{\partial z} \Delta_{j} f\left(g(t)+z g^{\prime}(t), g^{\prime}(t)+z g^{\prime \prime}(t), \ldots, g^{(j)}(t)+z g^{(j+1)}(t)\right)\right|_{z=0} \\
& =\Delta_{j+1} f\left(g(t), g^{\prime}(t), \ldots, g^{(j+1)}(t)\right) .
\end{aligned}
$$

The theorem follows by induction.

We can give an alternative expression for $\Delta_{n} f$ and hence for the chain rule (3) in terms of the elementary symmetric functions $\sigma_{k}$. If $z=\left(z_{1}, \ldots, z_{n}\right)$ then $\sigma_{k}(z)$ will denote the elementary symmetric function of degree $k$ in $z_{1}, \ldots, z_{n}$, so that, for all $\lambda \in \mathbb{C}$,

$$
\left(\lambda+z_{1}\right) \cdots\left(\lambda+z_{n}\right)=\lambda^{n}+\sigma_{1}(z) \lambda^{n-1}+\cdots+\sigma_{n}(z) .
$$


Lemma 1 Let $f: \Omega \subset X \rightarrow Y$ have $n$ continuous Fréchet derivatives, where $X, Y$ are Banach spaces and $\Omega$ is an open set. For any $x_{0} \in \Omega$ and $x_{1}, \ldots, x_{n} \in X$,

$$
\Delta_{n} f\left(x_{0}, x_{1}, \ldots, x_{n}\right)=\left.\frac{\partial^{n}}{\partial z_{n} \partial z_{n-1} \ldots \partial z_{1}} f\left(x_{0}+\sigma_{1}(z) x_{1}+\cdots+\sigma_{n}(z) x_{n}\right)\right|_{z=(0, \ldots, 0)} .
$$

Proof. The case $n=1$ is simple. Suppose that $n>1$ and that the expression holds for $n-1$. If $z^{\prime}=\left(z_{1}, \ldots, z_{n-1}\right)$ we have

$$
\begin{aligned}
\Delta_{n} f\left(x_{0}, x_{1}, \ldots, x_{n}\right)= & \left.\frac{\partial}{\partial z_{n}} \Delta_{n-1} f\left(x_{0}+z_{n} x_{1}, \ldots, x_{n-1}+z_{n} x_{n}\right)\right|_{z_{n}=0} \\
= & \frac{\partial}{\partial z_{n}} \frac{\partial^{n-1}}{\partial z_{n-1} \ldots \partial z_{1}} f\left(x_{0}+z_{n} x_{1}+\sigma_{1}\left(z^{\prime}\right)\left(x_{1}+z_{n} x_{2}\right)\right. \\
& \left.\quad+\cdots+\sigma_{n-1}\left(z^{\prime}\right)\left(x_{n-1}+z_{n} x_{n}\right)\right)\left.\right|_{z^{\prime}=(0, \ldots, 0), z_{n}=0}
\end{aligned}
$$

Since, for $0 \leq j \leq n-1$,

$$
\sigma_{j}\left(z_{1}, \ldots, z_{n-1}\right) z_{n}+\sigma_{j+1}\left(z_{1}, \ldots, z_{n-1}\right)=\sigma_{j+1}\left(z_{1}, \ldots, z_{n}\right),
$$

we have

$$
\Delta_{n} f\left(x_{0}, x_{1}, \ldots, x_{n}\right)=\left.\frac{\partial^{n}}{\partial z_{n} \partial z_{n-1} \ldots \partial z_{1}} f\left(x_{0}+\sigma_{1}(z) x_{1}+\cdots+\sigma_{n}(z) x_{n}\right)\right|_{z=(0, \ldots, 0)}
$$

as required.

Corollary 1 If $f, g$ are as in Theorem 2 then, for any $t \in \Psi$,

$$
(f \circ g)^{(n)}(t)=\left.\frac{\partial^{n}}{\partial z_{n} \partial z_{n-1} \ldots \partial z_{1}} f\left(g(t)+\sigma_{1}(z) g^{\prime}(t)+\cdots+\sigma_{n}(z) g^{(n)}(t)\right)\right|_{z=(0, \ldots, 0)} .
$$

The third chain rule applies to more general composite functions on Banach spaces.

Theorem 3 Let $W, X, Y$ be Banach spaces over $\mathbb{K}$ and let $\Phi, \Omega$ be open subsets of $W, X$ respectively. Let

$$
g: \Phi \rightarrow \Omega, \quad f: \Omega \rightarrow Y
$$

have $n$ continuous Fréchet derivatives, for some $n \in \mathbb{N}$. For any $w_{0} \in \Phi$ and $w_{1}, w_{2}, \ldots, w_{n} \in W$,

$$
\Delta_{n} f \circ g\left(w_{0}, w_{1}, \ldots, w_{n}\right)=\Delta_{n} f\left(g\left(w_{0}\right), \Delta_{1} g\left(w_{0}, w_{1}\right), \ldots, \Delta_{n} g\left(w_{0}, w_{1}, \ldots, w_{n}\right)\right) .
$$


Proof. Given any $w_{0} \in \Phi$ and $w_{1} \in W$, set $G(z):=g\left(w_{0}+z w_{1}\right)$ for $z$ in a neighbourhood $\Psi$ of 0 , so that $G: \Psi \subseteq \mathbb{K} \rightarrow \Omega$ is continuously differentiable at 0 with $G^{\prime}(0)=\Delta_{1} g\left(w_{0}, w_{1}\right)$. Then

$$
\Delta_{1} f \circ g\left(w_{0}, w_{1}\right)=\left.\frac{\partial}{\partial z} f\left(g\left(w_{0}+z w_{1}\right)\right)\right|_{z=0}=(f \circ G)^{\prime}(0) .
$$

By Theorem 2,

$$
(f \circ G)^{\prime}(0) .=\Delta_{1} f\left(G(0), G^{\prime}(0)\right)=\Delta_{1} f\left(g\left(w_{0}\right), \Delta_{1} g\left(w_{0}, w_{1}\right)\right) .
$$

Hence

$$
\Delta_{1} f \circ g\left(w_{0}, w_{1}\right)=\Delta_{1} f\left(g\left(w_{0}\right), \Delta_{1} g\left(w_{0}, w_{1}\right)\right),
$$

which proves the result in the case $n=1$.

Suppose the assertion holds for some $j<n$, that is, for any $w_{0} \in \Phi$ and $w_{1}, \ldots, w_{j} \in W$

$$
\Delta_{j}(f \circ g)\left(w_{0}, \ldots, w_{j}\right)=\Delta_{j} f\left(g\left(w_{0}\right), \ldots, \Delta_{j} g\left(w_{0}, \ldots, w_{j}\right)\right) .
$$

Let $w_{0} \in \Phi, w_{1}, \ldots, w_{j+1} \in W$ be given and, for $z$ in a neighbourhood $\Psi$ of 0 , set

$$
Z_{0}(z):=g\left(w_{0}+z w_{1}\right), \ldots, Z_{j}(z):=\Delta_{j} g\left(w_{0}+z w_{1}, \ldots, w_{j}+z w_{j+1}\right) .
$$

By the inductive hypothesis,

$$
\begin{aligned}
\Delta_{j+1}(f \circ g)\left(w_{0}, \ldots, w_{j+1}\right) & =\left.\frac{\partial}{\partial z} \Delta_{j}(f \circ g)\left(w_{0}+z w_{1}, \ldots, w_{j}+z w_{j+1}\right)\right|_{z=0} \\
& =\left.\frac{\partial}{\partial z} \Delta_{j} f\left(Z_{0}(z), \ldots, Z_{j}(z)\right)\right|_{z=0} .
\end{aligned}
$$

Define $G: \Psi \subseteq \mathbb{K} \rightarrow \Omega \times X^{j-1}$ by

$$
G(z):=\left(Z_{0}(z), \ldots, Z_{j}(z)\right),
$$

so that

$$
\Delta_{j+1}(f \circ g)\left(w_{0}, \ldots, w_{j+1}\right)=\left(\Delta_{j} f \circ G\right)^{\prime}(0) .
$$

Write

$$
\zeta_{0}=Z_{0}(0), \ldots, \zeta_{j}=Z_{j}(0)
$$

and

$$
\zeta_{j+1}=Z_{j}^{\prime}(0)
$$

Note that, for $1 \leq k \leq j+1$,

$$
\zeta_{k}=Z_{k-1}^{\prime}(0)=\Delta_{k} g\left(w_{0}, \ldots, w_{k}\right),
$$


whence $G$ is differentiable at 0 with

$$
G^{\prime}(0)=\left(\zeta_{1}, \ldots, \zeta_{j+1}\right)
$$

By equation (4) and Theorem 2,

$$
\begin{aligned}
\Delta_{j+1}(f \circ g)\left(w_{0}, \ldots, w_{j+1}\right) & =\left(\Delta_{j} f \circ G\right)^{\prime}(0)=\Delta_{1} \Delta_{j} f\left(G(0), G^{\prime}(0)\right) \\
& =\Delta_{1} \Delta_{j} f\left(\left(\zeta_{0}, \ldots, \zeta_{j}\right) ;\left(\zeta_{1}, \ldots, \zeta_{j+1}\right)\right) \\
& =\left.\frac{\partial}{\partial t} \Delta_{j} f\left(\zeta_{0}+t \zeta_{1}, \ldots, \zeta_{j}+t \zeta_{j+1}\right)\right|_{t=0} \\
& =\Delta_{j+1} f\left(\zeta_{0}, \ldots, \zeta_{j+1}\right) \\
& =\Delta_{j+1} f\left(g\left(w_{0}\right), \ldots, \Delta_{j+1} g\left(w_{0}, \ldots, w_{j+1}\right)\right) .
\end{aligned}
$$

Therefore, by induction, the formula holds for $n \in \mathbb{N}$.

Let us note some simple properties of higher directional derivatives.

Theorem 4 Let $X, Y$ be Banach spaces over $\mathbb{K}$, let $\Omega$ be an open set in $X$ and let $f$ : $\Omega \rightarrow Y$ have $n$ continuous Fréchet derivatives. For any $x_{0} \in \Omega, x_{1}, \ldots, x_{n}, \xi_{1}, \xi_{2} \in X$ and $\alpha \in \mathbb{K}$,

$$
\Delta_{n} f\left(x_{0}, \alpha x_{1}, \ldots, \alpha^{n} x_{n}\right)=\alpha^{n} \Delta_{n} f\left(x_{0}, x_{1}, \ldots, x_{n}\right)
$$

and

$$
\begin{array}{r}
\Delta_{n} f\left(x_{0}, \ldots, x_{n-1}, \alpha \xi_{1}+(1-\alpha) \xi_{2}\right)=\alpha \Delta_{n} f\left(x_{0}, \ldots, x_{n-1}, \xi_{1}\right) \\
+(1-\alpha) \Delta_{n} f\left(x_{0}, \ldots, x_{n-1}, \xi_{2}\right) .
\end{array}
$$

These properties follow simply by induction. Note that $\Delta_{n} f\left(x_{0}, x_{1}, \ldots, x_{n}\right)$ is affine in $x_{n}$. Second, a version of Leibniz' formula holds for $\Delta_{n}$.

Theorem 5 Let $W, X_{1}, X_{2}$ and $Y$ be Banach spaces, let $\Psi$ be an open subset of $W$ and let $B: X_{1} \times X_{2} \rightarrow Y$ be a continuous bilinear operator. Let $f: \Psi \rightarrow X_{1}$ and $g: \Psi \rightarrow X_{2}$ be $n$ times continuously Fréchet differentiable. Then, for any $\left(w_{0}, w_{1}, \ldots, w_{n}\right) \in \Psi \times W^{n}$,

$$
\begin{aligned}
\Delta_{n}(B \circ(f \times g))\left(w_{0}, w_{1}, \ldots, w_{n}\right) \\
=\sum_{m=0}^{n}\left(\begin{array}{c}
n \\
m
\end{array}\right) B\left(\Delta_{m} f\left(w_{0}, \ldots, w_{m}\right), \Delta_{n-m} g\left(w_{0}, \ldots, w_{n-m}\right)\right) .
\end{aligned}
$$

\section{Examples}

Three examples will suffice to illustrate the chain rule formulae in the preceding section. 
Example 2 - derivatives of $e^{g}$.

On combining Theorem 2 and Example 1 we find, for any $\mathcal{C}^{3}$ function $g$ and $t \in \mathbb{C}$,

$$
\frac{d^{3}}{d t^{3}} e^{g(t)}=\Delta_{3} \exp \left(g(t), g^{\prime}(t), g^{\prime \prime}(t), g^{\prime \prime \prime}(t)\right)=e^{g(t)}\left(g^{\prime}(t)^{3}+3 g^{\prime}(t) g^{\prime \prime}(t)+g^{\prime \prime \prime}(t)\right) .
$$

It is easy to see by induction that, for any $n \in \mathbb{N}$, there is a polynomial $Y_{n}$ of degree $n$ in $x_{1}, \ldots, x_{n}$ such that

$$
\Delta_{n} \exp \left(x_{0}, x_{1}, \ldots, x_{n}\right)=e^{x_{0}} Y_{n}\left(x_{1}, \ldots, x_{n}\right),
$$

and so that

$$
\frac{d^{n}}{d t^{n}} e^{g(t)}=e^{g(t)} Y_{n}\left(g^{\prime}(t), \ldots, g^{(n)}(t)\right)
$$

whenever $g$ is $n$ times continuously differentiable. The $Y_{n}$ can be derived recursively: $Y_{1}\left(x_{1}\right)=x_{1}$, and the relation

$$
e^{x_{0}} Y_{n+1}\left(x_{0}, \ldots, x_{n+1}\right)=\left.\frac{\partial}{\partial z} e^{x_{0}+z x_{1}} Y_{n}\left(x_{1}+z x_{2}, \ldots, x_{n}+z x_{n+1}\right)\right|_{z=0}
$$

translates into the recursion

$$
Y_{n+1}\left(x_{0}, \ldots, x_{n+1}\right)=x_{1} Y_{n}\left(x_{1}, \ldots, x_{n}\right)+\sum_{j=1}^{n} x_{j+1} \frac{\partial Y_{n}}{\partial x_{j}}\left(x_{1}, \ldots, x_{n}\right) .
$$

The polynomials $Y_{n}$ have a long history. They are called after Eric T. Bell who studied them in 1934 [1], but Johnson [8, Section 2] traces related polynomials to sundry 19th century authors, including Faà di Bruno himself. $Y_{n}$ can be defined combinatorially, or by means of the elegant determinantal formula (which apparently is essentially due to Faà di Bruno)

$$
Y_{n}\left(x_{1}, \ldots, x_{n}\right)=\left|\begin{array}{cccccc}
\left(\begin{array}{c}
n-1 \\
0
\end{array}\right) x_{1} & \left(\begin{array}{c}
n-1 \\
1
\end{array}\right) x_{2} & \left(\begin{array}{c}
n-1 \\
2
\end{array}\right) x_{3} & \ldots & \left(\begin{array}{c}
n-1 \\
n-2
\end{array}\right) x_{n-1} & \left(\begin{array}{c}
n-1 \\
n-1
\end{array}\right) x_{n} \\
-1 & \left(\begin{array}{c}
n-2 \\
0
\end{array}\right) x_{1} & \left(\begin{array}{c}
n-2 \\
1
\end{array}\right) x_{2} & \ldots & \left(\begin{array}{l}
n-2 \\
n-3
\end{array}\right) x_{n-2} & \left(\begin{array}{c}
n-2 \\
n-2
\end{array}\right) x_{n-1} \\
0 & -1 & \left(\begin{array}{c}
n-3 \\
n-3
\end{array}\right) x_{1} & \ldots & \left(\begin{array}{l}
n-3 \\
n-4
\end{array}\right) x_{n-3} & \left(\begin{array}{c}
n-3 \\
n-3
\end{array}\right) x_{n-2} \\
\vdots & \vdots & \vdots & & \vdots & \vdots \\
0 & 0 & 0 & \ldots & \left(\begin{array}{l}
1 \\
0
\end{array}\right) x_{1} & \left(\begin{array}{l}
1 \\
1
\end{array}\right) x_{2} \\
0 & 0 & 0 & \ldots & -1 & \left(\begin{array}{l}
0 \\
0
\end{array}\right) x_{1}
\end{array}\right| .
$$

Faà di Bruno's formula can be succinctly expressed in terms of the coefficients of the Bell polynomials $Y_{n}$ : for proofs and much interesting history see Johnson, loc. cit.. The purpose of this note is rather to exhibit some simpler forms of higher chain rules.

Example 3 Let us calculate the third derivative of $f\left(g_{1}(t), g_{2}(t)\right)$ for suitably smooth 
functions $f$ on $\mathbb{R}^{2}, g_{j}$ on $\mathbb{R}$. For $x_{j}, y_{j} \in \mathbb{R}$ we find

$$
\begin{aligned}
\Delta_{1} f\left(\left(x_{0}, y_{0}\right),\left(x_{1}, y_{1}\right)\right) & =\left(x_{1} \frac{\partial}{\partial x}+y_{1} \frac{\partial}{\partial y}\right) f\left(x_{0}, y_{0}\right) \\
\Delta_{2} f\left(\left(x_{0}, y_{0}\right),\left(x_{1}, y_{1}\right),\right. & \left.\left(x_{2}, y_{2}\right)\right)=\left(\left(x_{2} \frac{\partial}{\partial x}+y_{2} \frac{\partial}{\partial y}\right) f\right. \\
\Delta_{3} f\left(\left(x_{0}, y_{0}\right),\left(x_{1}, y_{1}\right),\right. & \left.\left.\left.\left(x_{2}, y_{2}\right),\left(x_{3}, y_{3}\right)\right)+y_{1} \frac{\partial}{\partial y}\right)^{2} f\right)\left(x_{0}, y_{0}\right) \\
= & \left(\left(x_{3} \frac{\partial}{\partial x}+y_{3} \frac{\partial}{\partial y}\right) f+3\left(x_{2} \frac{\partial}{\partial x}+y_{2} \frac{\partial}{\partial y}\right)\left(x_{1} \frac{\partial}{\partial x}+y_{1} \frac{\partial}{\partial y}\right) f\right. \\
& \left.+\left(x_{1} \frac{\partial}{\partial x}+y_{1} \frac{\partial}{\partial y}\right)^{3} f\right)\left(x_{0}, y_{0}\right) .
\end{aligned}
$$

Hence

$$
\begin{aligned}
\frac{d^{3}}{d t^{3}} f\left(g_{1}(t), g_{2}(t)\right)= & \Delta_{3} f\left(\left(g_{1}(t), g_{2}(t)\right), \ldots,\left(g_{1}^{\prime \prime \prime}(t), g_{2}^{\prime \prime \prime}(t)\right)\right) \\
= & \left(\left(g_{1}^{\prime \prime \prime}(t) \frac{\partial}{\partial x}+g_{2}^{\prime \prime \prime}(t) \frac{\partial}{\partial y}\right) f\right. \\
& +3\left(g_{1}^{\prime \prime}(t) \frac{\partial}{\partial x}+g_{2}^{\prime \prime}(t) \frac{\partial}{\partial y}\right)\left(g_{1}^{\prime}(t) \frac{\partial}{\partial x}+g_{2}^{\prime}(t) \frac{\partial}{\partial y}\right) f \\
& \left.\quad+\left(g_{1}^{\prime}(t) \frac{\partial}{\partial x}+g_{2}^{\prime}(t) \frac{\partial}{\partial y}\right)^{3} f\right)\left(g_{1}(t), g_{2}(t)\right) .
\end{aligned}
$$

More generally, one can express $\Delta_{n} f$ in terms of higher-order Fréchet derivatives of $f$, e.g.,

$$
\Delta_{3} f\left(x_{0}, x_{1}, x_{2}, x_{3}\right)=D f\left(x_{0}\right)\left(x_{3}\right)+3 D^{2} f\left(x_{0}\right)\left(x_{1}, x_{2}\right)+D^{3} f\left(x_{0}\right)\left(x_{1}, x_{1}, x_{1}\right) .
$$

In view of Theorem 2 , it is clear that a similarly explicit expression for $\Delta_{n} f$ would be equivalent to a version of the Faà di Bruno formula; indeed, from equation (1) (or its multivariate generalization) we must have

$$
\Delta_{n} f\left(x_{0}, \ldots, x_{n}\right)=\sum \frac{n !}{k_{1} \ldots ! k_{n} !(1 !)^{k_{1}} \ldots(n !)^{k_{n}}} D^{k} f\left(x_{0}\right)\left(x_{1}, \ldots, x_{1}, \ldots, x_{n}, \ldots, x_{n}\right)
$$

where, on the right-hand side, the argument $x_{j}$ is repeated $k_{j}$ times, and the sum is taken over all nonnegative integer solutions for the $k_{j}$ of the Diophantine equation $k_{1}+2 k_{2}+\cdots+n k_{n}=n$, and $k:=k_{1}+\cdots+k_{n}$. An independent proof of this formula, together with Theorem 2, would yield yet another proof of the Faà di Bruno formula.

Here is a multivariate example.

Example 4 Let $f_{m}: \mathbb{C}^{k \times k} \rightarrow \mathbb{C}$ be defined by $f_{m}(X)=\operatorname{tr}^{2}\left(X^{m}\right)$, and let us calculate $\Delta_{2} f_{m}\left(X_{0}, X_{1}, X_{2}\right)$. Write $p_{m}(X)=X^{m}$. By Theorem 3 we have

$$
\begin{aligned}
\Delta_{2} f_{m}\left(X_{0}, X_{1}, X_{2}\right) & =\Delta_{2}\left(\operatorname{tr}^{2} \circ p_{m}\right)\left(X_{0}, X_{1}, X_{2}\right) \\
& =\Delta_{2} \operatorname{tr}^{2}\left(p_{m}\left(X_{0}\right), \Delta_{1} p_{m}\left(X_{0}, X_{1}\right), \Delta_{2} p_{m}\left(X_{0}, X_{1}, X_{2}\right)\right) .
\end{aligned}
$$


On one hand,

$$
\begin{aligned}
\Delta_{1} \operatorname{tr}^{2}\left(X_{0}, X_{1}\right) & =2 \operatorname{tr} X_{0} \operatorname{tr} X_{1}, \\
\Delta_{2} \operatorname{tr}^{2}\left(X_{0}, X_{1}, X_{2}\right) & =2 \operatorname{tr}^{2} X_{1}+2 \operatorname{tr} X_{0} \operatorname{tr} X_{2} .
\end{aligned}
$$

On the other hand,

$$
\begin{aligned}
\Delta_{1} p_{m}\left(X_{0}, X_{1}\right) & =\left.\frac{\partial}{\partial z}\left(X_{0}+z X_{1}\right)^{m}\right|_{z=0}=\sum_{\substack{\alpha, \beta \geq 0 \\
\alpha+\beta=m-1}} X_{0}^{\alpha} X_{1} X_{0}^{\beta}, \\
\Delta_{2} p_{m}\left(X_{0}, X_{1}, X_{2}\right) & =\left.\frac{\partial}{\partial z} \sum_{\substack{\alpha, \beta \geq 0 \\
\alpha+\beta=m-1}}\left(X_{0}+z X_{1}\right)^{\alpha}\left(X_{1}+z X_{2}\right)\left(X_{0}+z X_{1}\right)^{\beta}\right|_{z=0} \\
& =\sum_{\substack{\alpha, \beta \geq 0 \\
\alpha+\beta=m-1}} X_{0}^{\alpha} X_{2} X_{0}^{\beta}+\sum_{\substack{\alpha, \beta, \gamma \geq 0 \\
\alpha+\beta+\gamma=m-2}} X_{0}^{\alpha} X_{1} X_{0}^{\beta} X_{1} X_{0}^{\gamma} .
\end{aligned}
$$

Therefore

$$
\begin{aligned}
& \Delta_{2} f_{m}\left(X_{0}, X_{1}, X_{2}\right)=2 \operatorname{tr}^{2} \Delta_{1} p_{m}\left(X_{0}, X_{1}\right)+2 \operatorname{tr} p_{m}\left(X_{0}\right) \operatorname{tr} \Delta_{2} p_{m}\left(X_{0}, X_{1}, X_{2}\right) \\
&=2 m^{2} \operatorname{tr}^{2}\left(X_{0}^{m-1} X_{1}\right) \\
&+2 \operatorname{tr}\left(X_{0}^{m}\right) \times\left\{m \operatorname{tr}\left(X_{0}^{m-1} X_{2}\right)+\sum_{\beta=0}^{m-2}(m-1-\beta) \operatorname{tr}\left(X_{0}^{m-2-\beta} X_{1} X_{0}^{\beta} X_{1}\right)\right\} .
\end{aligned}
$$

\section{Acknowledgements}

The first and second named authors gratefully acknowledge the hospitality of the School of Mathematics and Statistics of the University of Newcastle upon Tyne.

\section{References}

[1] E. T. Bell, Exponential polynomials, Ann. of Math. (2) 35 (1934) 258-277.

[2] G. M. Constantine and T. H. Savits, A multivariate Faà di Bruno formula with applications, Transactions of the American Mathematical Society 348(2) (1996) 503-520.

[3] Cav. F. Faà di Bruno, Sullo sviluppo delle funzioni, Annali di Scienze Matematiche e Fisiche 6 (1855) 479-480.

[4] Cav. F. Faà di Bruno, Note sur une nouvelle formule de calcul différentiel, Quarterly J. Pure Appl. Math. 1 (1857) 359-360.

[5] http://www-groups.des.st-and.ac.uk/ history/Mathematicians/Faa_di_Bruno. html. 
[6] H.-N. Huang, S.A.M. Marcantognini, and N. J. Young, The spectral Carathéodory-Fejér problem, to appear in Integr. equ. oper. theory

[7] I. L. Iribarren, Calculo Diferencial en Espacios Normados, Equinoccio, 1980.

[8] W. P. Johnson, The curious history of Faà di Bruno's formula, American Mathematical Monthly 109(2002) 217-234.

[9] R. L. Mishkov, Generalization of the formula of Faà di Bruno for a composite function with a vector argument, Internat. J. Math. Sci. 24(7) (2000) 481-491.

[10] J.D. DePree, and C.W. Swartz, Introduction to Real Analysis, John Wiley \& Sons, Inc., New York, 1998.

[11] K. Spindler, A short proof of the formula of Faà di Bruno, Elemente der Mathematik 60 (2005), 33-35.

\section{H.-N. Huang}

Department of Mathematics, Tunghai University, Taichung 40704, Taiwan.

nhuang@mail.thu.edu.tw

\section{S.A.M. Marcantognini}

Department of Mathematics, Instituto Venezolano de Investigaciones Cientificas, P.O. Box 21827, Caracas 1020A, Venezuela.

smarcant@ivic.ve

\section{N. J. Young}

School of Mathematics and Statistics, University of Newcastle upon Tyne, NE1 7RU, England.

N.J.Young@ncl.ac.uk 